\title{
A young boy with unusual foot lump (madura foot)
}

\author{
S. Rajendra, M. Sivashankar \\ Department of Surgery, University of Jaffna, Sri Lanka.
}

Keywords: Madura foot; eumycetoma; acinomycetoma; fungal grain; diagnostic management

\section{Introduction}

Mycetoma is a debilitating gradually progressing chronic infectious disease affecting skin and subcutaneous tissue. Gill first described this condition in India as "Madura Foot" in 1842. Carter initiated using the terminology "Mycetoma" in 1860.

Mycetoma when caused by true fungi it is known as eumycetoma and that is caused by microaerophilic actinomycetes is known as actinomycetoma. Geographical distribution of Mycetoma reveals endemic pattern in tropical and subtropical areas [1]. It remains as a real cause of disability among population living in rural agricultural area as it is often neglected in the initial stage. Early identification and intervention gives good result.

\section{Case report}

18 years old boy presented with large lump in his right side sole for three years duration. It was which progressively increasing in size, with associated yellow colour fluid discharge from the surface of lump. On examination there was a large $10 \times 15 \mathrm{~cm}$ irregular protruding growth over anterior two thirds of the sole which was indurated and has multiple nodular areas with sinus tracts (Figure 1).

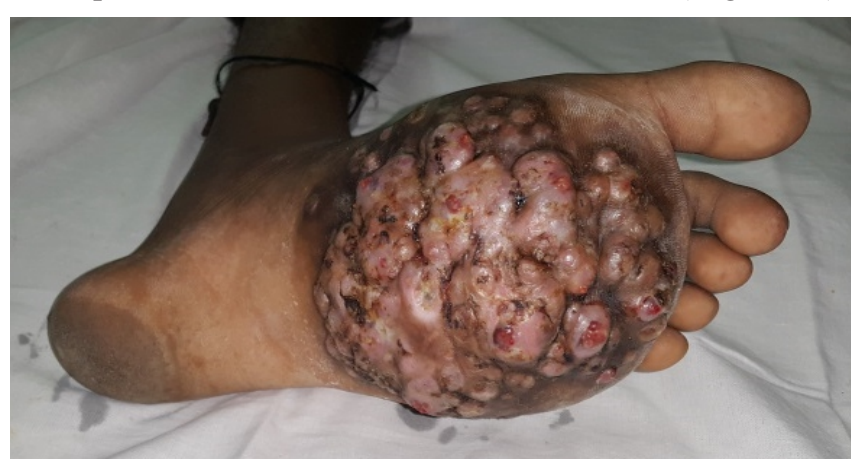

Figure 1. Myecetoma involving the anterior half of sole in right

Correspondence: S. Rajendra

E-mail: dr.s.rajendra@gmail.com

Received: 28-01-2017 Accepted: 02-04-2017

(iD) $h$ ttp://orcid.org/0000-0002-3303-603X

DOI: http://doi.org/10.4038/sljs.v35i1.8355 (cc)BY

The Sri Lanka Journal of Surgery 2017; 35(1): 37-39
The excision biopsy from the lesion showed chronic inflammatory process with multiple granules in tissues, which supported the diagnosis of mycetoma infection.

MRI scan revealed a $10 \mathrm{~cm}$ x $8 \mathrm{~cm}$ large soft tissue mass in sole of right foot with "dot in circle" sign and osteomyelitis changes in $2^{\text {nd }}$ to $4^{\text {th }}$ metatarsal bones (Figure 2).

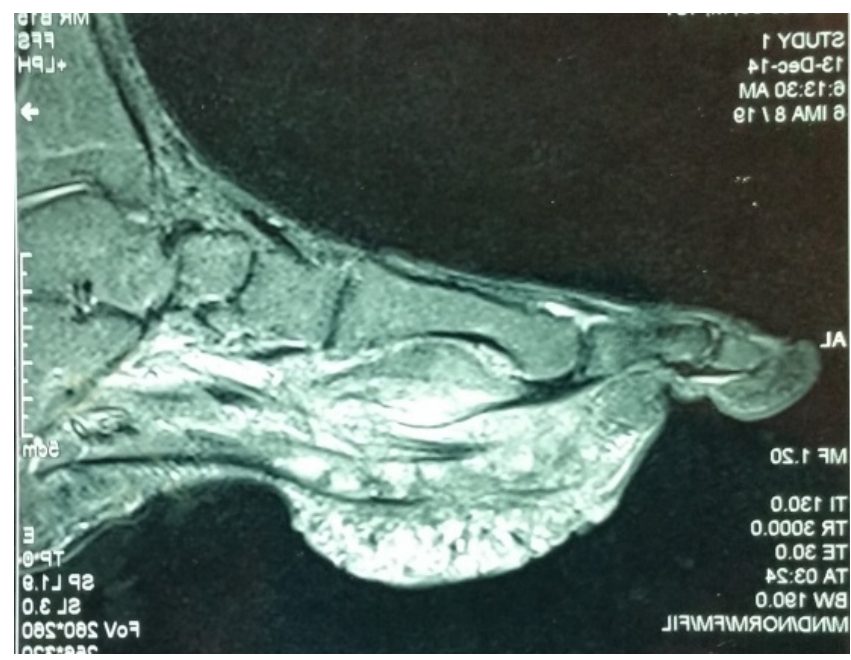

Figure 2. The MRI scan shows the soft tissue lump in sole suggestive of Madura foot

There were no abnormalities in routine blood investigations. He was treated with antifungal drugs (itraconazole and cotrimoxazole) for three months duration as advised by dermatologist. One month after commencing the treatment, a wide local excision of lump was carried out in collaboration with plastic surgical team.

The histology specimen showed the chronic inflammatory areas with central granules exhibiting radiating filamentous materials. These granules were rimmed by neutrophils and foreign body type giant cells. These features were compatible with a clinical diagnosis of Madura foot.

H\&E staining appearance could result from infections due to mycetoma infection. A skin graft was performed to the excision site and the patient had a good healing (Figure 3).

The patient was then given the remaining course of treatment and was advised to take long term with itrconazole. 


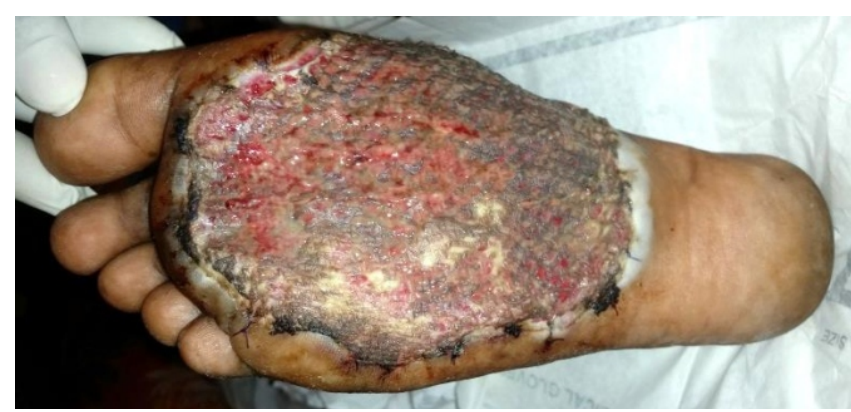

Figure 3. Picture shows skin graft after excision of lump

\section{Discussion and conclusions}

$40 \%$ of Mycetoma infection is due to eumycetoma. 30 different species are known to cause mycetoma infection. Actinomadura madurae has been the most common causative species among actinomycetoma. Madurella mycetomatis has been the most common causative species among eumycetoma and is an important infective species globally [1]

Although Mycetoma can affect patients at any age yet the peak age incidence lies between the age 20 and 40 . Foot infection by Mycetoma is the most common site (68.7\%) of infection in human body. Pathogens get access to skin from soil via superficial abrasions or lacerations [1]. The incubation period for the Mycetoma infection is around three months [4]. Agricultural workers, farmers and those who don't use footwear are prone to get infection. Males are affected more than females (male to female ratio of 5:1) probably because of the occupational exposure [1].

The clinical triad characterizing Mycetoma infection are chronic induration (subcutaneous mass), draining sinuses, and discharge of granules [1]. The disease can be staged from A to $\mathrm{D}$ based on the worsening clinical manifestations. When there is swelling with no sinuses it would be stage A and the development of woody induration with sinuses is stage $\mathrm{B}$. Bone involvement is stage $C$ and the spread to other sites and multiple lesions is stage D [4]. There are reports about the remote possibility of regional lymph node spread [1].

Different types of imaging modalities used to identify the extent of tissue involvement. Based on the X-ray findings the extent of Mycetoma infection has been staged from 0 to stage VI ( 0 -no bone involvement and stage VI - multiple rays and multiple bone involvement) [5]. Mycetoma Skin, Muscle, Bone Grading System (MSMBS), is an MRI diagnosis of extent of Mycetoma infection in respective tissues [6].

Grains varying in physical properties could be demonstrated from the sinuses. They can be identified by haematoxylin and eosin (H\&E) staining and can aid at identification of organism. A Gram stain is useful for detecting actinomycetoma and Periodic acid-Schiff (PAS) staining is useful to detect eumycetoma [3].
Culture methods are the gold standard to identify species of mycetoma responsible for infection. It is time consuming and can also have contamination. Further some species can't be identified by morphology alone [1]. Appropriate molecular diagnostic procedures would aid to identify the causative agent of Mycetoma. A specific PCR test was designed to amplify a region of ribosomal gene complex. Species identification becomes possible with post amplification restriction digestion technique [1].

Treatment type and response differ between actinomycetoma and eumycetoma. As such it is a prerequisite to identify the correct species for effective treatment. Eumycetoma is better treated with adequate antifungal therapy and surgery. Actinomycetoma usually responds well to antibiotics and surgery will be needed in few selected cases. The best outcome could be expected for an early well encapsulated lesion without bone involvement, by combining medical treatment before and after surgery [1].

The treatment of advanced mycetoma infection usually needs long term antimicrobial treatment. The side effects of drugs and the possibility of relapse of infection will have to be borne in mind during follow up [1].

\section{References}

1. Ahmed W, Fahal A, van de Sande W, Verbrugh H: Mycetoma caused by Madurellamycetomatis; Lancet Infectious Diseases (2004), 566-574.

https://doi.org/10.1016/S1473-3099(04)01131-4

2. Maiti PK, Ray A, Bandyopadhyay S. Epidemiological aspects of mycetoma from a retrospective study of 264 cases in West Bengal. Tropical Medicine \& International Health. 2002 Sep 1;7(9):788-92.

https://doi.org/10.1046/j.1365-3156.2002.00915.x

3. van de Sande WW, Fahal AH, Goodfellow M, Welsh O, Zijlstra EE. Merits and pitfalls of currently used diagnostic tools in mycetoma. PLoS Negl Trop Dis. 2014 Jul 3;8(7):e2918. https://doi.org/10.1371/journal.pntd.0002918

4. Maina AM, Macharia JT. Alleviating a Nomad's Anguish: Successful Treatment of a Case of Leg Mycetoma-A Case Report. Case reports in orthopedics. 2012 Dec 3;2012.

5. Abd El Bagi ME. New radiographic classification of bone involvement in pedal mycetoma. American Journal of Roentgenology. 2003 Mar;180(3):665-8. https://doi.org/10.2214/ajr.180.3.1800665

6. Shamy ME, Fahal AH, Shakir MY, Homeida MM. New MRI grading system for the diagnosis and management of mycetoma. Transactions of the Royal Society of Tropical Medicine and Hygiene. 2012 Dec 1;106(12):738-42. https://doi.org/10.1016/j.trstmh.2012.08.009 


\section{Key Points:}

- Madura foot is caused by fungus or actinomycosis infection.

- It's a chronic granulomatous infection, characterized by "tumour like" indurations in skin with sinuses discharging pus, blood and grains.

- Cases identified early could be effectively treated with antifungal or antimicrobials. If untreated at early stages it will present with debilitating massive tumour like lesions.

- Advanced cases of Madura foot in extremities may end up in surgical amputation. 